\title{
Harmonic Detection System and Identification Algorithm for Steel Pipeline Defects
}

\author{
Yizhen Zhao ${ }^{1}$, Xinhua Wang ${ }^{1 *}$, Mingfei Wang ${ }^{2}$, Yu Duan ${ }^{1}$, Lin Yang $^{1}$, Qingfeng Pan ${ }^{1}$, Xuyun Yang ${ }^{3}$ \\ ${ }^{1}$ Faculty of Materials and Manufacturing, College of Mechanical Engineering and Applied Electronics Technology, Beijing \\ University of Technology, Beijing 100124, China \\ ${ }^{2}$ National Research Center of Intelligent Equipment for Agriculture, Beijing 100097, China \\ ${ }^{3}$ China Special Equipment Inspection and Research Institute, Beijing 100013, China
}

Corresponding Author Email: paper_bgdjd103@163.com

https://doi.org/10.18280/ejee.230103

Received: 2 August 2020

Accepted: 14 January 2021

\section{Keywords:}

harmonic excitation, multichannel acquisition, defects detection, target identification

\begin{abstract}
Aiming at the problem of defects detection of steel pipeline, a harmonic detection system was developed based on electromagnetic principle, and the target signal identification algorithm was studied. The Advanced RISC Machine (ARM) Cortex-M3 was adopted to design digital adjustable harmonic excitation source, and its effective output power can up to $70 \mathrm{~W}$. The Field Programmable Gate Arrays (FPGA) and ARM Cortex-M4 were introduced to design 15 channels high speed data collector, which parallel local-storage rate of each channel can reach $4.7 \mathrm{kHz}$. The electromagnetic focusing excitation array and Tunnel Magneto Resistance (TMR) sensors array were constructed to improve the spatial resolution of the detection system. Meanwhile, the system also integrated GPS positioning and LCD real-time display functions. Furthermore, the algorithm combining Empirical Mode Decomposition (EMD) and variable-scale Stochastic Resonance (SR) was proposed to process signal and enhance the targets. The effectiveness of the instrument and algorithm are well verified in both simulation and experiment. The results show that this method has higher integration and better detection effect, which provides a novel method for non-contact detection of metal material defects and is suitable for engineering applications.
\end{abstract}

\section{INTRODUCTION}

As one of the important oil and gas transportation modes, pipeline transportation plays an extremely important role in national economy and human daily life. More than half of the oil and gas transportation in the world is realized by pipeline, with long mileage and long service life. During operation, due to the effects of corrosion, man-made damage, natural disasters and other factors, some defects will occur inevitably, which will cause the leakage of the medium. Especially in the case of heating and pressurizing the medium, it is prone to cause fire, explosion and other accidents when leakage occurs. It can also cause serious environmental pollution, heavy casualties and economic losses. Therefore, in order to avoid the occurrence of malignant pipeline accidents, it is necessary to carry out damage detection on pipelines regularly.

At present, several commonly used traditional NonDestructive Testing (NDT) methods include X-ray testing [1], ultrasonic testing [2, 3], magnetic particle testing [4], penetration testing [5], and eddy current testing [6, 7]. However, radiation is harmful to the human body; ultrasonic testing requires coupling agent; magnetic particle testing and penetration testing need surface cleaning and pretreatment; eddy current testing is limited by skin effect. In addition, the smart Pig based on Magnetic Flux Leakage (MFL) can also realize the on-line detection of pipeline defects [8], but the pig crawling in the pipeline is easily limited by space and angle, which often causes congestion or loss, and the cost is very high. Transient Electromagnetic Method (TEM) can detect defects such as wall thickness thinning and pipe body crack [9]. However, due to the characteristics of attenuation and discontinuity, it still needs further research and improvement. Magnetic Tomography Method (MTM) and Geomagnetic Method (GM) are passive detection technologies based on environmental magnetic field [10,11], which are easy to operate and can reflect the stress concentration area. But the passive magnetic field is very weak and the anti-interference ability is poor, which makes the detection signal vulnerable to external interference, difficult to extract effective information, low detection rate and poor accuracy. NoPig [12] detection equipment is large and complex to operate; Ultrasonic Guided Wave Testing (UGWT) [13] detection distance is short, and the results tend to overlap. Through the above analysis, an excellent pipeline detection should have the following characteristics: high signal strength, strong penetration capability, non-contact online detection, high acquisition efficiency, high resolution, high equipment integration, simple operation, etc.

Therefore, it is very important to propose a novel pipeline defect detection method and develop a special detection instrument in order to better meet the practical engineering applications. We actively apply the multi-frequency excitation signal to pipeline under the non-contact condition, and then the array sensors acquire the signal and extracts the magnetic anomaly target at the defect. The magnetic field measurement sensors usually include the following types: Hall Effect sensor, Fluxgate sensor, Anisotropic Magneto Resistance (AMR) sensor, Giant Magneto Resistance (GMR) sensor, Tunnel 
Magneto Resistance (TMR) sensor, Superconducting Quantum Interference Device (SQUID), and etc. [14-19]. Among them, the TMR sensor is based on electron spin characteristics and quantum tunnel effect, which has low power consumption and high sensitivity. It has the advantages of AMR and GMR sensors, and its cost is lower than quantum sensors. In addition, the existing detection instruments are mostly composed of several parts, such as industrial computers and data acquisition cards [20], which are bulky and complicated to operate. With the development of embedded hardware, the integration degree of instruments is getting higher and higher. The high performance CPU is widely used, such as ARM, DSP and FPGA can overcome the bottleneck of hardware development and improve the running speed [21-24], which can provide ideas for the development of pipeline defects detection instrument.

The actual detected signal will be polluted by noise inevitably, which makes the defect signal very weak. Bosse et al. [25] designed the sensors array and Multiple Signal Classification (MUSIC) algorithm to identify the target signal source to realize the defect location in the plane metallic structure. Huang et al. [26] used Empirical Mode Decomposition (EMD) algorithm to decompose and reconstruct the collected signals, which can recognize the porosity defects in the aluminum alloy weld. Song et al. [27] introduced Wavelet Transform (WT) to process the magnetic field signal component and identified the crack defects of the high-pressure pipe. Different from the traditional filtering algorithm, Wan et al. [28] used the stochastic resonance (SR) algorithm to transfer the noise energy and realize the extraction of the magnetic anomaly target. Therefore, in order to realize the identification of pipeline defects, it is necessary to propose an effective target signal extraction algorithm.

In this paper, a harmonic detection method for pipeline defects is proposed. This method combines high frequency and ultra-low frequency sine continuous electromagnetic wave to generate harmonic excitation signal, and uses the TMR sensors array to record the echo signal. A dedicated detection instrument was developed based on a high-performance ARM and FPGA dual CPUs. Finally, the EMD and SR algorithms are introduced to identify the target signal, which can realize the defects detection of the pipeline wall surface and subsurface.

\section{HARMONIC EXCITATION AND FOCUSING ARRAY}

The steel pipeline detection method based on harmonic principle can enhance the target signal strength effectively at the pipeline defects by applying the excitation signal actively. At the same time, it can shield the background noise and external interference to a certain extent. In addition, the composition of the excitation signal and the form of the coils array are related to the intensity of the excitation signal acting on the defect targets of the pipeline.

\subsection{Development of excitation source}

The Micro-programmed Control Unit (MCU) of ARM Cortex-M3 is used as the core controller of harmonic excitation source, and the Frequency Modulated Carrier Wave (FMCW) [29] function is realized based on Direct Digital Synthesis (DDS). After signal synthesis, the excitation coils array is driven by the power amplifier circuit directly, and the harmonic parameters are displayed in the 3.5 inch LCD. The frequency and power of the excitation source are adjustable, which can achieve accurate and stable harmonic signal. In order to facilitate the application in practical engineering, the $12 \mathrm{~V} \mathrm{Li-Battery}$ supplies power for system. The overall architecture of the hardware is shown in Figure 1.
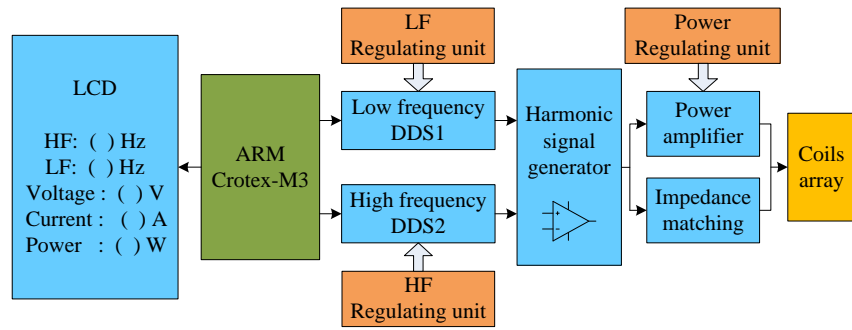

Figure 1. Architecture of excitation source hardware

Because of the electromagnetic shielding on the metal pipe wall, the ultra-low frequency electromagnetic wave is introduced into the excitation source as the carrier signal, and the high frequency detection signal is carried at the same time. A programmable DDS chip AD9833 is used to generate a single wave, which includes a precision reference clock, a phase accumulator and register, a Digital-to-Analog Converter (DAC) and a low-pass filter. It can generate sine wave, triangle wave and square wave, and the output frequency range is $0 \mathrm{~Hz}$ to $12.5 \mathrm{MHz}$. AD9833 is connected to the digital potentiometer MCP41010 and adjusted by the program in the MCU through Serial Peripheral Interface (SPI) communication. However, the output voltage of the chip is about 0 to $650 \mathrm{mV}$. Therefore, AD8051 is used to design the in-phase proportional operation amplifier circuit, and its output signal is amplified as shown in Figure 2.

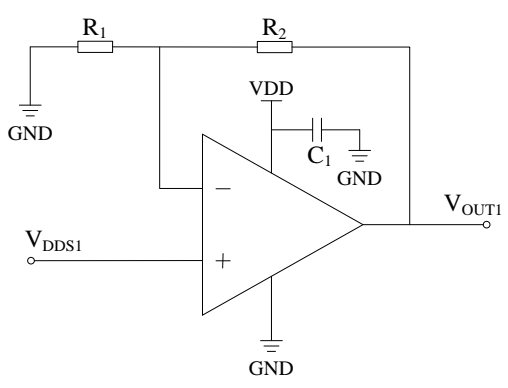

Figure 2. Signal amplifying circuit

In this circuit, the in-phase input of amplifier is the sinusoidal signal from the DDS output, and the anti-phase input is the signal after dividing the amplifier output value by resistors R1 and R2. According to the "virtual short" feature of the amplifier, the two input terminals have the same voltage without considering the influence of leakage current. It is possible to amplify the input signal $\mathrm{V}_{\mathrm{DDS}}$ to a certain multiple by adjusting the values of resistors $\mathrm{R} 1$ and $\mathrm{R} 2$. The amplifier model can be expressed as:

$$
V_{\text {OUT1 }}=V_{D D S 1} \times\left(1+\frac{R_{2}}{R_{1}}\right)
$$

In this paper, DDS is set as a sinusoidal signal with the initial phase of 0 , the frequency is adjustable by pressing the 
key, and the amplitude is adjusted by feedback resistor. Similarly, the same circuit can generate another sinusoidal signal. Based on the principle of FMCW, the harmonic generation circuit is designed. LM324 operational amplifier is used to realize DDS signal synthesis with different frequency by constructing the add operation circuit. The mathematical analytical model can be expressed as:

$$
f(t)=\sum_{i=0}^{n} f_{i}(t)=\sum_{i=0}^{n} A_{i} \sin \left(2 \pi f_{i} t+\theta_{i}\right)
$$

where, $i$ is the number of sine wave; $A_{i}$ is the amplitude; $f_{i}$ is the frequency component; $\theta_{i}$ is the initial phase angle. Then, a cascade voltage follower is designed to improve the input impedance and reduce the output impedance, while acting as a buffering isolator to improve the circuit robustness. It is similar to the in-phase proportional op-amp circuit, but the voltage follower feeds back all the output voltage to the antiphase input terminal so that the feedback factor is 1 . The harmonic synthesis signal is shown in Figure 3.

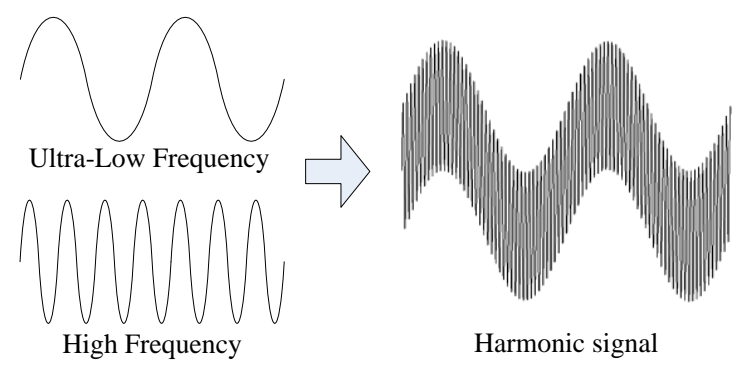

Figure 3. Harmonic synthesis

Harmonic wave does not have the ability to drive the load, it can only be as a signal source. In order to make the harmonic excitation signal meet the requirements of detecting pipeline defects, the coils array is driven by high voltage and high current operational amplifier OPA549. With DC boost module, $12 \mathrm{~V}$ DC power supply is converted to $\pm 30 \mathrm{~V}$ to provide "rail to rail" external voltage for amplifier. Then, the in-phase proportional operational amplifier circuit and the voltage follower circuit are designed to improve the harmonic amplification accuracy and drive capability. The harmonic output power is adjusted by the slide rheostat in the negative feedback circuit.

In order to meet the parameter setting of harmonic excitation source in different detection requirements, the harmonic parameters are displayed in LCD, including the high frequency (HF) component and low frequency (LF) component of harmonic, as well as the actual driving voltage, current and power. Among them, the frequency of harmonic components can be obtained and displayed directly when DDS is generated, but other parameters acquisition requires the external detection circuit. A precise detection circuit of True Root Mean Square (TRMS) of harmonic signal is designed, which uses the amplification and depth negative feedback of LMC6482 to reduce the non-linearity of diode and the error effect of threshold voltage. Then, the conversion from TRMS to $\mathrm{DC}$ is realized by AD637, and the second-order low-pass filter is realized by OPA177 precision operational amplifier, with a cut-off frequency of $10 \mathrm{~Hz}$. High precision current transformer with serial port is used to obtain the output current, and the baud rate is set to $9600 \mathrm{bps}$.
Under the premise of a certain output impedance, the maximum effective output power of the harmonic excitation source can be more than $70 \mathrm{~W}$, and the actual hardware is shown in Figure 4.

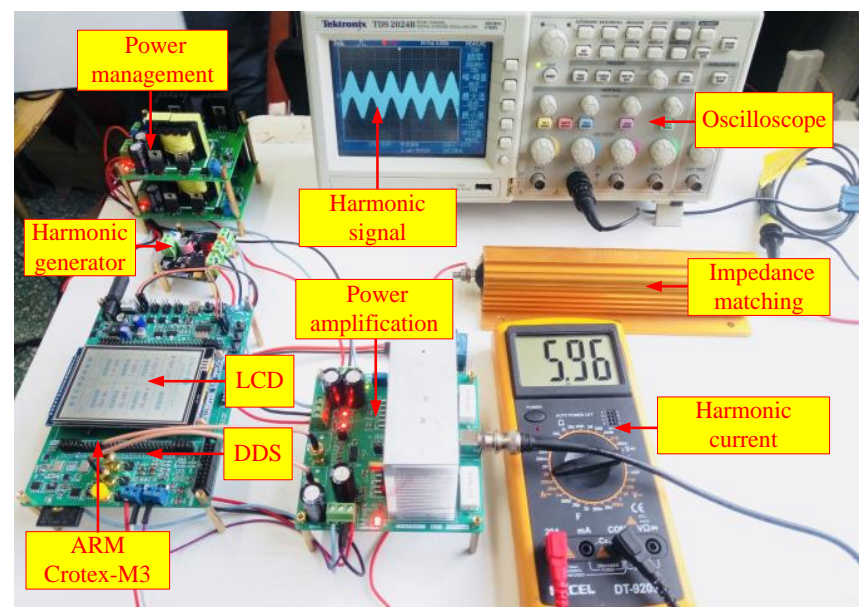

Figure 4. Harmonic excitation source test

\subsection{Design of focusing array detector}

After impedance matching, the harmonic signal is sent to the exciting coils array directly, but the lift-off distance will lead to the intensity attenuation of the excitation signal. So the array detector focusing structure is proposed to improve the actual detection effect of harmonic excitation. Using $0.5 \mathrm{~mm}$ diameter enamelled copper wire, five square excitation coils are made, and each of them has 20 turns. Without considering the coils width, four small square coils with side length of $20 \mathrm{~cm}$, one large square coil with side length of $43 \mathrm{~cm}$, and the distance between each coil set as $1 \mathrm{~cm}$, are made to form a focus array in this paper. According to the vector signal characteristics of harmonic magnetic field, the TMR threedimensional (3D) vector sensor is used to replace the traditional detection coil. The detector structure is shown in Figure 5.

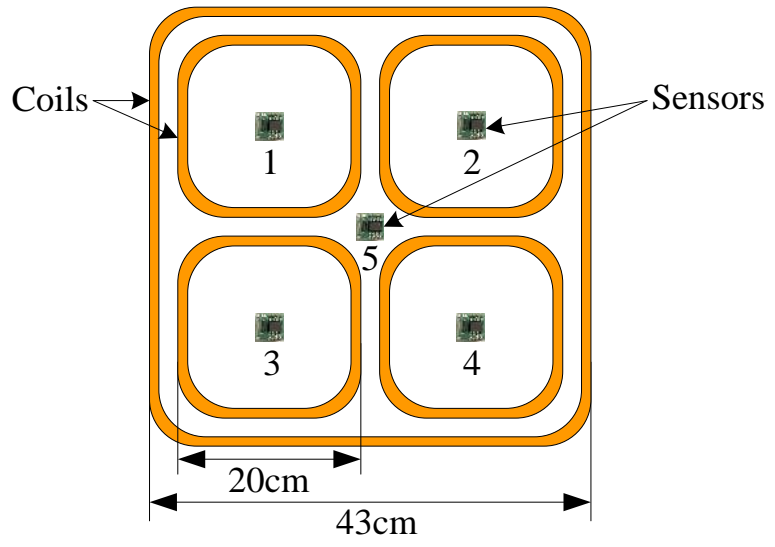

Figure 5. The detector structure

Based on the tunnel magneto resistance effect, the harmonic magnetic field is collected. The signal of each axis of the sensor is output in the form of difference, which has higher stability and anti-interference ability. The detector is more sensitive to pipeline small defects, and obtains higher detection sensitivity and spatial resolution, breaking through 
the half wavelength limit. At the same time, the TMR array expands the coverage of the received signal and provides the feasibility for the detection of the pipeline side defects.

During the operation of the system, the 5-element array coils are applied with harmonic excitation signals in parallel, and five TMR vector sensors collect the harmonic echo signals. The array detector designed in this paper is analysed by finite element. When the harmonic current is set to $2 \mathrm{~A}$ and the lifting distance is $100 \mathrm{~cm}$, the focusing effect is as shown in Figure 6.

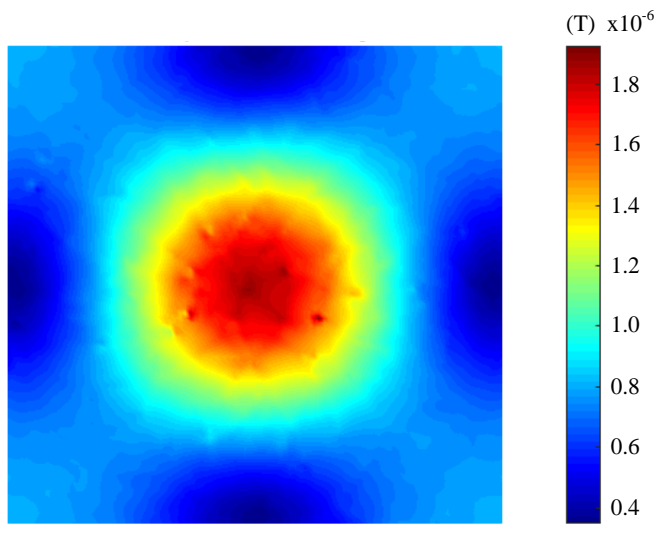

Figure 6. Array focusing

The three-dimensional Cartesian coordinate system is established with the center of the array as the origin, and the array and non-array excitation probes are compared under different lift off distances. Within the range of $[-50,50] \mathrm{cm}$ detection line below the detector, the lift-off height is set at 30 $\mathrm{cm}, 50 \mathrm{~cm}$ and $70 \mathrm{~cm}$ respectively, and the harmonic flux density mode gradually decreases. The performance of the excitation focusing array is about 2 times higher than the nonarray detector. Under the same excitation intensity, the effective excitation coverage area is larger, which enhances the effect of pipeline damage detection, as shown in Figure 7.

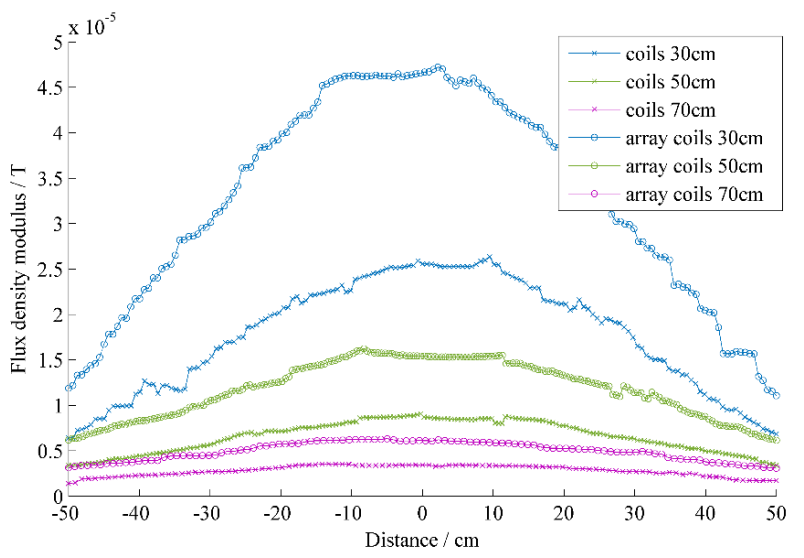

Figure 7. Focusing performance comparison

\section{HIGH SPEED PARALLEL HARMONIC ACQUISITION AND STORAGE}

The differential TMR 3D vector sensor array improves the detection ability, but puts forward higher requirements for the acquisition and storage of multi-channel sensor data. FPGA (EP4CE10E22C8) and ARM Cortex-M4 (STM32F407) dual controllers system is adopted [30]. The front-end controls the parallel acquisition of 15 channel harmonic signals, system time and XYZ three-axis curve display. The back-end realizes the USB flash disk data storage, GPS positioning, key and LED functions. The system architecture is shown in Figure 8.

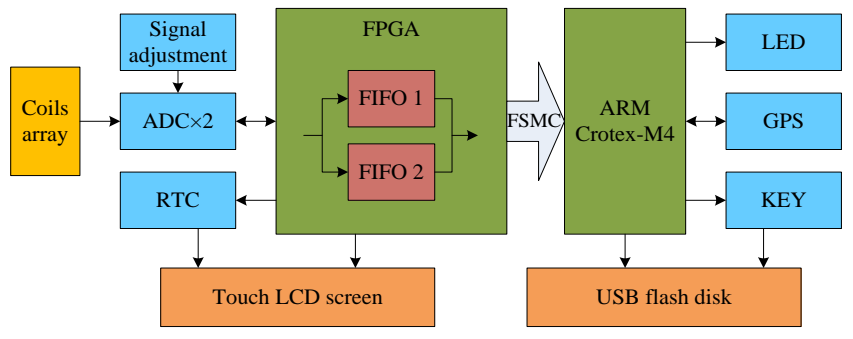

Figure 8. Architecture of high speed acquisition system

\subsection{FPGA - signal acquisition}

TMR sensor is composed of three unique push-pull Wheatstone full bridge, in which each axis signal is output in the form of differential analog voltage. The array detector has five sensors, including 30 channels of analog voltage signal. In order to save ADC resources, eight AD8277 integrated operational amplifier differential subtraction circuits control the conversion from differential voltage to single terminal voltage signal. The integrated operational amplifier has high gain accuracy, and the common mode rejection ratio (CMRR) can reach $86 \mathrm{~dB}$, which can realize the precise adjustment of 15 -axis harmonic signal. In addition, because of the positive and negative value of the harmonic magnetic field collected by the sensor, the AD8277 needs bipolar power supply. MAX1626 and LM2662 are used to supply 5V and -5V power respectively.

AD7606 is an integrated 8-channel and 16bit high-precision synchronous sampling Analog-to-Digital Converter (ADC), which can acquire \pm 5 and \pm 10 true bipolar input analog signals with $+5 \mathrm{~V}$ single power supply. It has a high input impedance of $1 \mathrm{M} \mathrm{Ohm}$, an on-chip anti-alias filter of $3 \mathrm{~dB}$ and a cut-off frequency of $22 \mathrm{kHz}$. In order to realize the high-speed acquisition of 15 channels harmonic signals, two AD7606 chips are adopted. The ADC controller is designed in FPGA, and the binary complement is calculated as the actual voltage value. The $30 \mathrm{MHz}$ clock is generated by Phase Locking Loop (PLL) in FPGA, which has high harmonic acquisition speed and clock frequency, but it is not suitable for subsequent data flow control. For the problem of clock mismatch, a 16bit asynchronous double First In First Out (FIFO) ping-pong structure is designed. When writing to FIFO1, it will automatically switch to read to FIFO2. When writing to FIFO2, it will switch to read to FIFO1 and poll in turn. Among them, the write clock is generated by FPGA and the read clock is generated by ARM Cortex-M4, which realizes the efficient transmission of data flow across the clock domain. It prevents data overflow and channel disorder.

FPGA displays the XYZ three-axis curve of the sensor in the center of the array in real-time in LCD. At the same time, the system depends on the Real Time Clock (RTC) chip DS1302 to provide the date and time, and an external passive crystal oscillator with frequency of $32.768 \mathrm{KHz}$ to provide the precise clock source. The chip is controlled by FPGA to communicate with SPI bus and displayed in LCD. Among them, the LCD is a 7-inch touch screen, and its Viewtech Graphical User Software (VGUS) configuration function 
greatly improves the development speed. The interface is designed in the computer, and the final configuration file can be imported into the LCD directly. In addition, the touchpad is designed with three touch command buttons: zoom in, zoom out and exit. The LCD interface is shown in Figure 9.

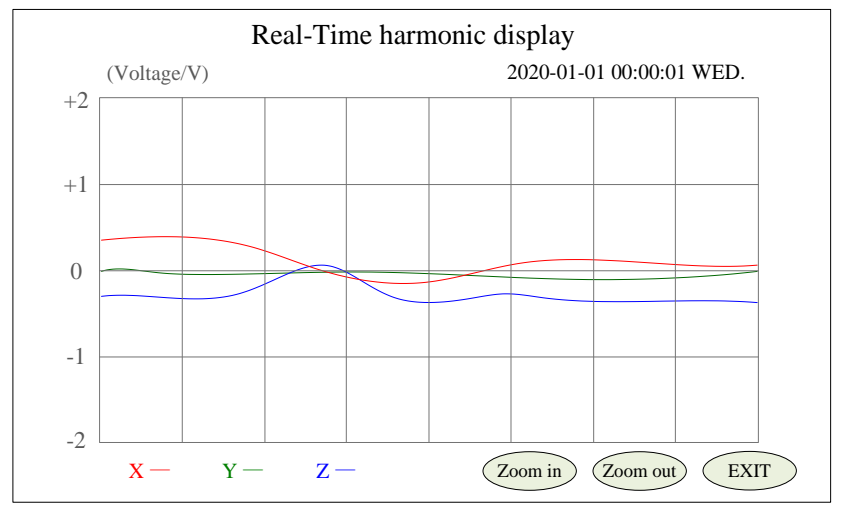

Figure 9. Touch screen interface

\subsection{ARM - signal storage}

In the practical application of engineering detection, it is necessary to ensure both the detection speed and the accuracy, so sufficient sampling rate is needed. Taking the artificial detection as an example, the moving speed is about $1 \mathrm{~m} / \mathrm{s}$, and the high frequency component of the harmonic is $1 \mathrm{kHz}$. According to Nyquist Theory, the sampling rate greater than $2 \mathrm{kHz}$ must be stored locally to ensure the complete analysis of subsequent signals. Under this condition, the resolution of pipeline detection within $0.5 \mathrm{~cm}$ can be ensured.

In order to meet the actual detection requirements, the USB flash disk with $32 \mathrm{G}$ capacity is used as the data storage medium, which has the characteristics of stability, plug and play. ARM Cortex-M4 has the function of On-The-Go (OTG), which is developed on the basis of USB2.0 protocol. It gets rid of the limitation of computer and realizes the data exchange of mobile terminal without host. The collected data points are stored in the size of $6 \times 8$ bit, the first bit is the symbol, and the last five bits are the data. For example, $-1.2345 \mathrm{v}$, the storage format is -12345 . The sensor array has 15 channels of data, each of those is stored at a synchronous storage rate of more than $2 \mathrm{kHz}$, with a total storage rate of at least $30 \mathrm{kHz}$ of signed data points. ARM Cortex-M4 controls the Flexible Static Memory Controller (FSMC) bus to read the data cached in the FIFO of FPGA and store it in the USB flash disk. The file format is $\mathrm{CSV}$, in which each column contains data for one channel, with a total of 15 columns. The name of created file is the date and time obtained in RTC module, so as to ensure that data files will not be duplicated or overwritten.

Most of the pipeline detection work is in outdoor environment, GPS positioning can help to determine the location of pipeline damage. The format of geographic coordinate information acquired by GPS module is NEMA0183, and its acquisition frequency is set to $5 \mathrm{~Hz}$. According to the longitude and latitude coordinates, the pipeline detection route is calculated. Combined with the detection environment and different external interferences, the detected pipeline sections are divided in detail. The collected signals in the corresponding pipeline sections are processed separately to ensure the effective location of the pipeline defects.

In addition, three LED indicators are designed. When the system is powered on, LED1 flashes at $1 \mathrm{~Hz}$ to prompt to wait for the USB flash disk to be inserted. When the USB flash disk is successfully tested and ready, LED1 is always on. LED2 blinks at $1 \mathrm{~Hz}$, indicating that the data is being stored. LED3 is to show system failure, and the system will automatically perform reset operation, otherwise it will be normally closed. Set the key interrupt function to control the stop of the acquisition system and the closing and saving of the CSV data file. After the execution, LED1 and LED2 will flicker at $2 \mathrm{~Hz}$ at the same time, indicating that the USB flash disk can be pulled out.

After testing the performance of the instrument, it is finally determined that the synchronous acquisition and storage rate of 15 channels can reach $4.7 \mathrm{KHz}$. The total storage rate of signed data points can reach more than $55 \mathrm{KHz}$ with stable and reliable performance. The actual hardware is shown in Figure 10.

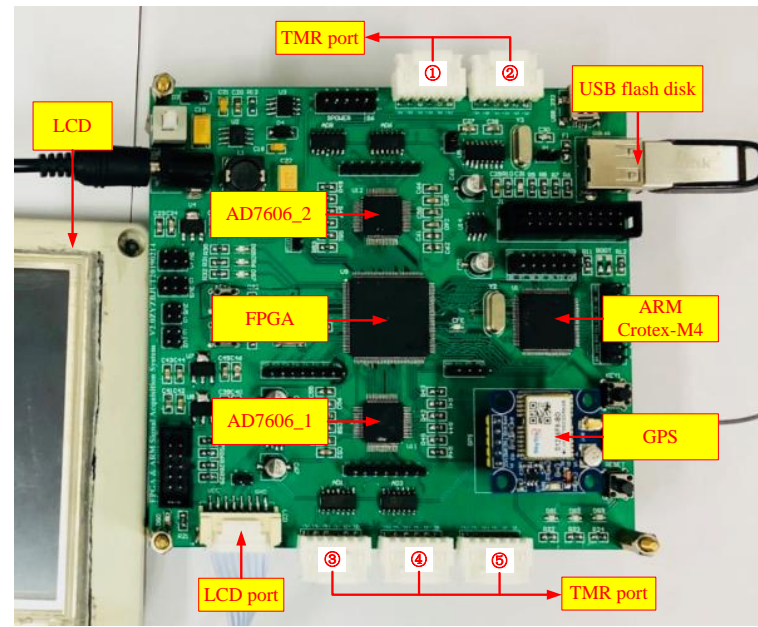

Figure 10. High speed acquisition system test

\section{DEFECTS ALGORITHM}

SIGNAL

IDENTIFICATION

In general, the environment and working condition of pipeline operation are complex, and there will be inevitably noise pollution in the collected signal, which brings great difficulties to the effective identification and extraction of pipeline defects signal. Under the background of strong noise and interference, the algorithm of EMD and SR is introduced to extract the target signal of pipeline defects.

\subsection{Empirical mode decomposition}

Empirical mode decomposition is an adaptive signal analysis algorithm without preset parameters, which can decompose signals into a series of Intrinsic Mode Functions (IMF) and residuals according to their own characteristics [31]

For signal $x(t)$, the steps of EMD algorithm can be divided as follows:

(1) Find the local maximum and minimum of the signal, and then the cubic spline method is adopted to fit the upper envelope $l_{\max }(t)$ and the lower envelope $l_{\min }(t)$;

(2) Calculate the average value of the upper and lower envelope, which can be defined as:

$$
m(t)=\frac{l_{\max }(t)+l_{\min }(t)}{2}
$$


The difference $c_{1}(t)$ between the original signal $x(t)$ and the average value $m(t)$ is regarded as an IMF to be selected, written as:

$$
c_{1}(t)=x(t)-m(t)
$$

(3) If $c_{1}(t)$ meets the IMF condition, set $d_{1}(t)=c_{1}(t)$, otherwise repeat steps (1) and (2) until $c_{1 k}(t)$ satisfies the IMF condition, where $k$ is the number of iterations.

(4) Remove the high frequency component and replace $x(t)$ with $r(t)$, expressed as:

$$
r_{1}(t)=x(t)-d_{1}(t)
$$

And repeat steps (1), (2), and (3) until the next IMF is obtained. When the EMD iteration is completed, the signal is decomposed into the following:

$$
x(t)=\sum_{i=1}^{I} d_{i}(t)+r_{I}(t)
$$

According to the above analysis, EMD can decompose complex signals adaptively. The simulation signal $x(t)$ is shown in Eq. (7), which contains two defects and the target signal is submerged completely by adding noise.

$$
x(t)=A_{1} \sin \left(2 \pi f_{1} t+\theta_{1}\right)+A_{2} \sin \left(2 \pi f_{2} t+\theta_{2}\right)
$$

Analyze the simulation signal and make Fast Fourier Transform (FFT), as shown in Figures 11(a) and (b).

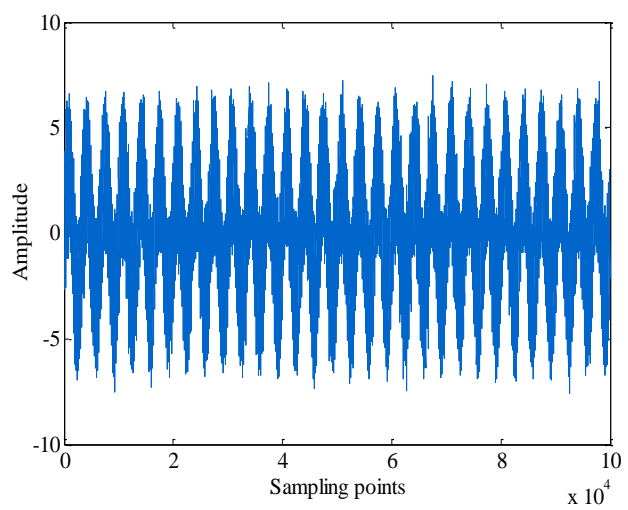

(a)

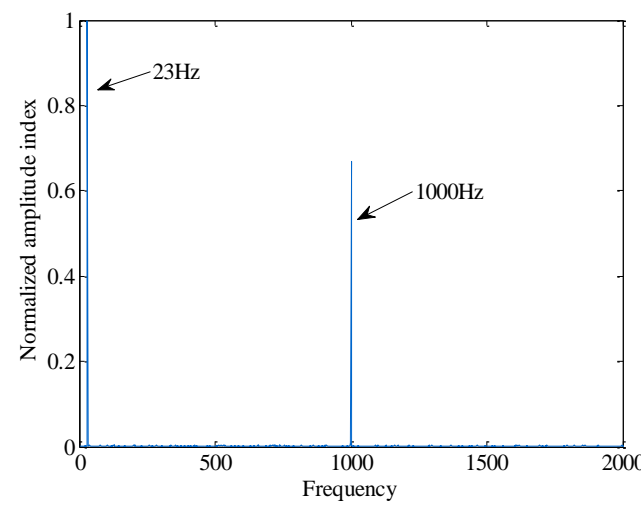

(b)

Figure 11. (a) Original signal submerged by noise. (b) Spectrum of signal
For the pipeline simulation signals with defects, the parameters are set as follows: $A_{1}=2, A_{2}=2.5, f_{1}=23 \mathrm{~Hz}$, $f_{2}=1000 \mathrm{~Hz}, \theta_{1}=\pi / 6, \theta_{2}=\pi / 3$.

After decomposition by EMD algorithm, the residual component is removed. Then the effective IMF components are selected and the square sum is calculated for time domain reconstruction. The targets signal are extracted and normalized as shown in Figure 12.

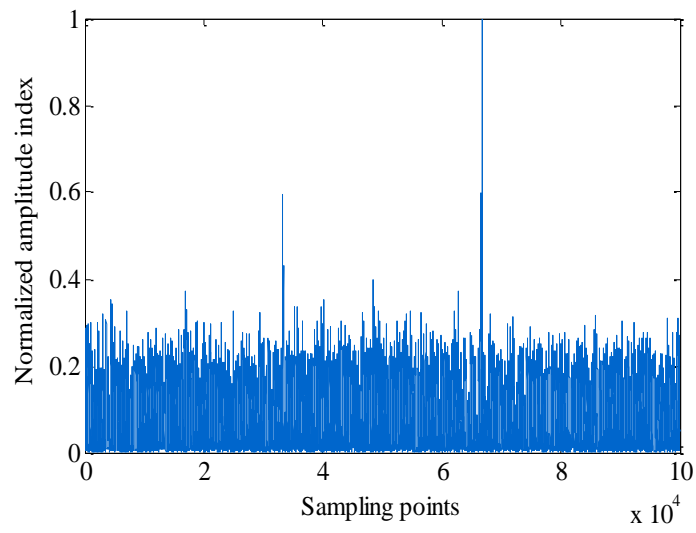

Figure 12. Target signal extraction by EMD algorithm

\subsection{Variable scale stochastic resonance}

Although EMD has the advantages of binary filter banks, it is prone to mode aliasing when dealing with complex signals with noise and mutation. Especially in the case of low Signal to Noise Ratio (SNR), modal aliasing seriously affects the performance of targets signal extraction. Based on the mechanism of energy transfer, SR makes the system, signal and noise achieve cooperation and produce resonance peaks. It is suitable for weak signal extraction and detection under strong noise background, so as to improve the targets extraction performance [32, 33].

The nonlinear bistable SR system can be represented by Langevin model, as follows:

$$
\frac{d x}{d t}=-\frac{d U(x)}{d t}+s(t)+n(t)
$$

where, $x(t)$ is the output signal of system; $s(t)$ is periodic or aperiodic signal; $n(t)$ is noise; $U(t)$ is the potential function of bistable system, which can be expressed as:

$$
U(x)=-\frac{a}{2} x^{2}+\frac{b}{4} x^{4}
$$

where, $a$ and $b$ are non-negative system parameters.

By adjusting the parameters of the system, the barrier height and the width of the potential well can be changed, which affects the transition ability of the particles. The parameters in the optimal output state can be obtained by judging the optimization conditions, so that the system can reach the state of stochastic resonance. The adjustment of the potential function is shown in Figures 13(a) and (b). It can be seen from the Figure 13 that the larger the parameter $a$ or the smaller the parameter $b$, the wider the potential well and the higher the potential barrier. 


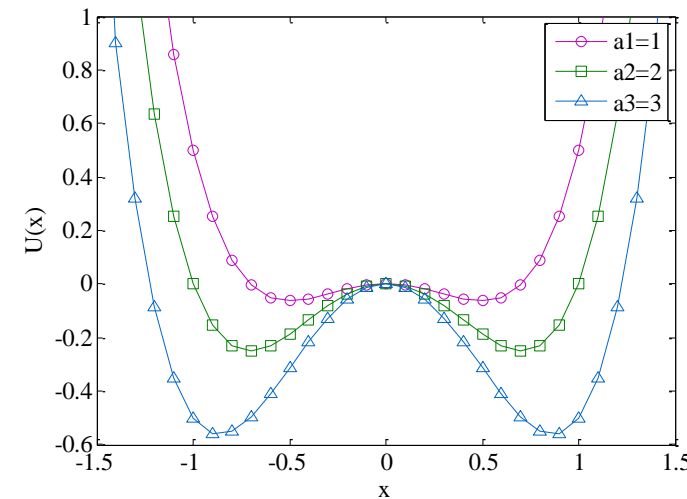

(a)

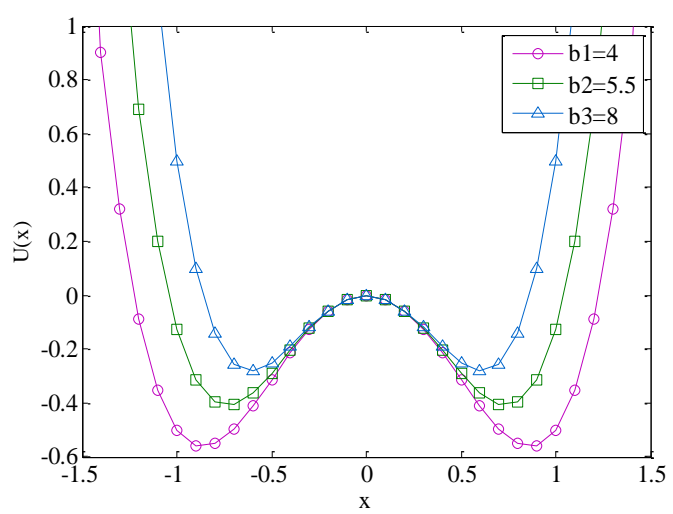

(b)

Figure 13. The change of potential function under different parameter settings. (a) $b=4, a_{1}=1, a_{2}=2, a_{3}=3$; (b)

$$
a=3, b_{1}=4, b_{2}=5.5, b_{3}=8 \text {. }
$$

Because of the large sampling frequency, it is necessary to change the scale to compress the signal frequency linearly to make it conform to the small parameter signal characteristics of the stochastic resonance system. The collected signals are all mixed signals with noise, which can be expressed as:

$$
s n(t)=s(t)+n(t)
$$

The SR model system can be solved by the fourth-order Runge-Kutta method, which is described as Eq. (11):

$$
\left\{\begin{array}{l}
x_{n+1}=x_{n}+\frac{h}{6}\left(K_{1}+2 K_{2}+2 K_{3}+K_{4}\right) \\
K_{1}=a x_{n}-b x_{n}^{3}+s n_{n} \\
K_{2}=a\left(x_{n}+\frac{K_{1}}{2}\right)-b\left(x_{n}+\frac{K_{1}}{2}\right)^{3}+s n_{n} \\
K_{3}=a\left(x_{n}+\frac{K_{2}}{2}\right)-b\left(x_{n}+\frac{K_{2}}{2}\right)^{3}+s n_{n+1} \\
K_{4}=a\left(x_{n}+K_{3}\right)-b\left(x_{n}+K_{3}\right)^{3}+s n_{n+1}
\end{array}\right.
$$

where, $h$ is the iteration step size, $x_{n}$ is the output signal of the SR system, $a$ and $b$ are system parameters.

After being processed by EMD algorithm, the signal is input to SR system for target enhancement, which will cause distortion in time domain. The time-domain recovery system is introduced to calculate its response signal, as shown in Figure 14.

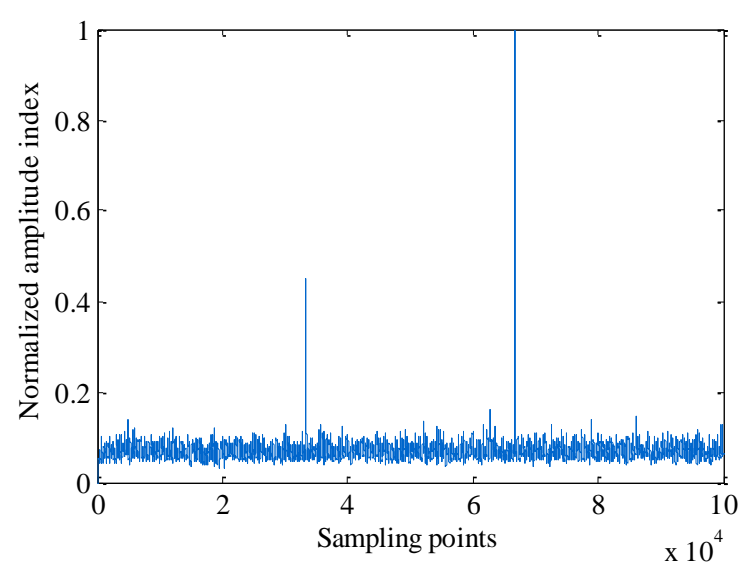

Figure 14. Response signal of SR recovery system

The algorithm of EMD and Variable Scale SR can extract the targets signal of pipeline defects effectively. The signal-tonoise ratio (SNR), root mean square error (RMSE) and kurtosis factor (KF) are used to evaluate the performance of the signal analysis method, as shown in Table 1.

Table 1. Algorithm performance evaluation

\begin{tabular}{cccc}
\hline & SNR & RMSE & KF \\
\hline EMD & 0.1052 & 2.6433 & 93.9715 \\
EMD and SR & 4.3592 & 0.0702 & 449.7216 \\
\hline
\end{tabular}

\section{EXPERIMENTAL VERIFICATION AND RESULTS}

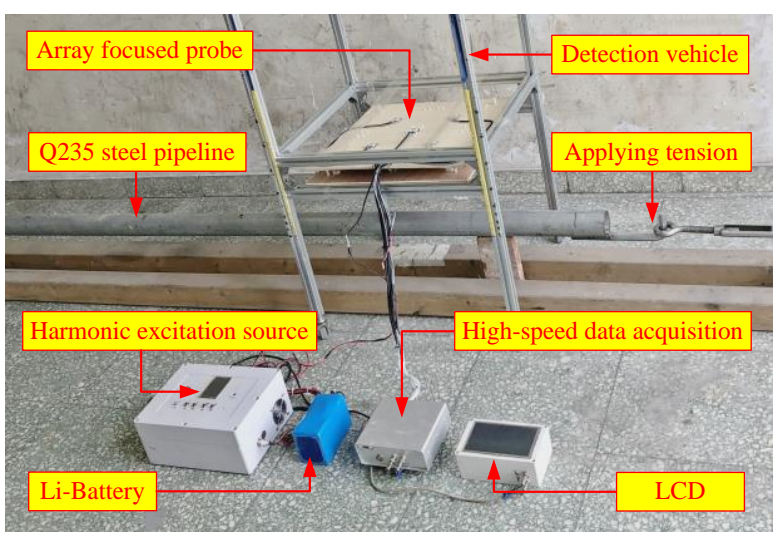

Figure 15. Experimental platform

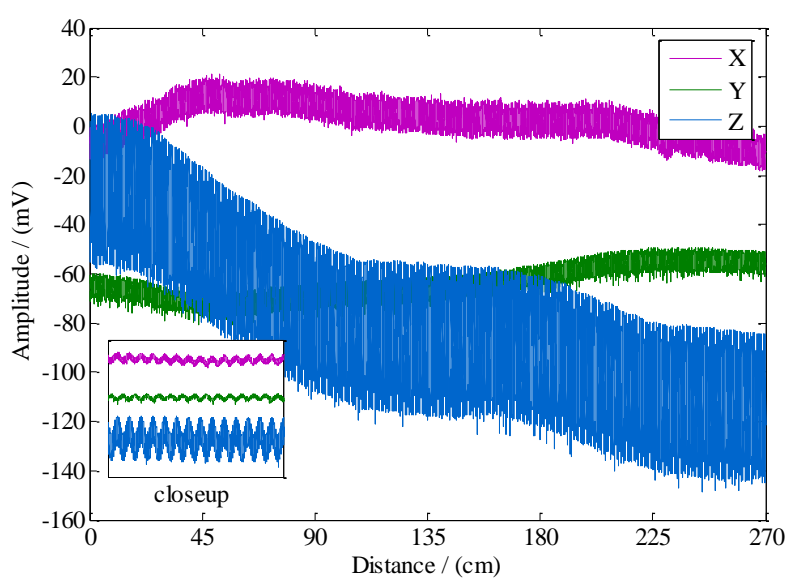

(a) 


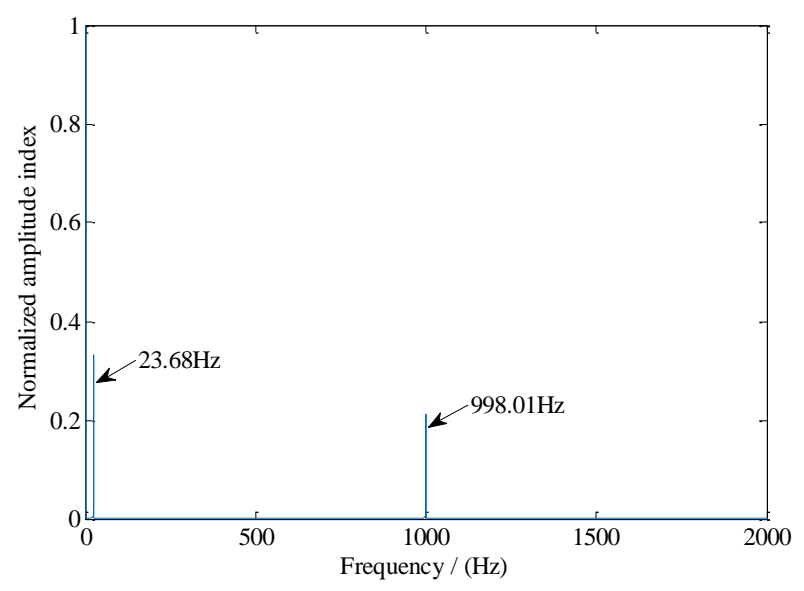

(b)

Figure 16. (a) The signal collected in the actual experiment; (b) Actual spectrum of signal

In order to verify the practicability of the harmonic excitation source and high-speed acquisition system, an indoor pipeline damage detection experimental platform is built. The developed system device is used for signal excitation and acquisition, and the target signal is extracted by the algorithm proposed in this paper. Q235 seamless steel pipeline is selected as the experimental pipeline, with a total length of 265 $\mathrm{cm}$, an outer diameter of $7.5 \mathrm{~cm}$ and a wall thickness of $0.4 \mathrm{~cm}$. There are six prefabricated defects on the pipe wall, which are axial crack, circumferential crack, rectangular groove, circular through hole, circular blind hole and 45-degree crack in turn, corresponding to the actual distances of $53 \mathrm{~cm}, 75 \mathrm{~cm}, 105 \mathrm{~cm}$, $144 \mathrm{~cm}, 185 \mathrm{~cm}$ and $217 \mathrm{~cm}$ respectively. In this experiment, the pipeline was placed horizontally and tension was applied on both sides. The harmonic excitation source is set as the empirical value of $23 \mathrm{~Hz}$ and $1000 \mathrm{~Hz}$, the sampling frequency of signal acquisition is set to $4.5 \mathrm{kHz}$, the lifting distance between the sensor and the pipeline is $30 \mathrm{~cm}$, and the detection vehicle keeps moving forward $270 \mathrm{~cm}$ at a constant speed. However, if the actual detection is performed outdoors or under other conditions, several people are needed to hold the probe and device. The indoor experiment platform is shown in Figure 15.

During the operation of the instrument, it can be seen from the LCD of the excitation source that the parameters of the harmonic signal are stable, and it can be seen from the LCD of the acquisition instrument that the echo signal recorded by the sensor array is also stable. After the experiment, the data is stored in the USB flash disk in the form of CSV. The data in the USB disk is exported for further processing to identify the pipeline defects. Take the data of No.5 TMR sensor as an example, XYZ three-axis signal is shown in Figure 16(a), and its FFT is shown in Figure 16(b).

The essence of the pipeline defect harmonic detection method is the identification of the magnetic anomaly target signal at the defect, which has the characteristics that the normal component crosses the zero point and the tangential component has the maximum value. Each single-axis signal is decomposed using EMD to obtain IMF and residual component, and select effective signal components to reconstruct and normalize the square sum. Then the signal is input into the variable scale SR system, and the appropriate parameter value is adjusted to make the system reach the optimal resonance output state and recover in time domain. The data processing flow is shown in Figure 17.

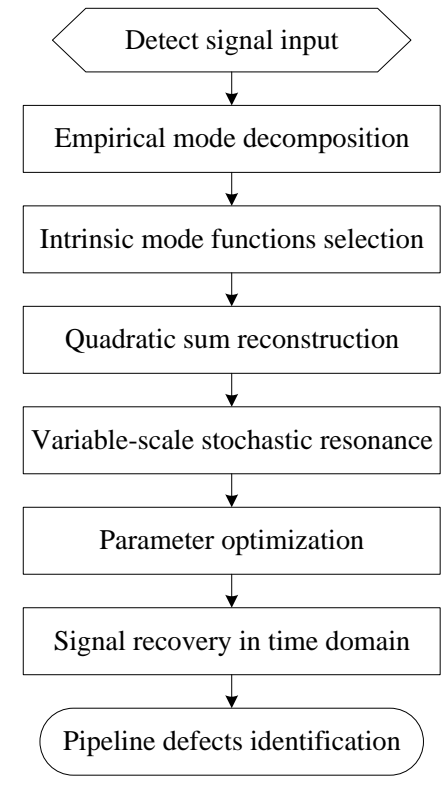

Figure 17. Data processing flow

Due to the different sensitivity of different defects signal to $\mathrm{XYZ}$ three-axis direction, 3D fusion of array signals is more effective for pipeline defects signal extraction. Comparing the signal processing results with the actual pipeline defects location, it is found that the experimental results are consistent with the expected target, which shows that different defects can be identified qualitatively, as shown in Figure 18.

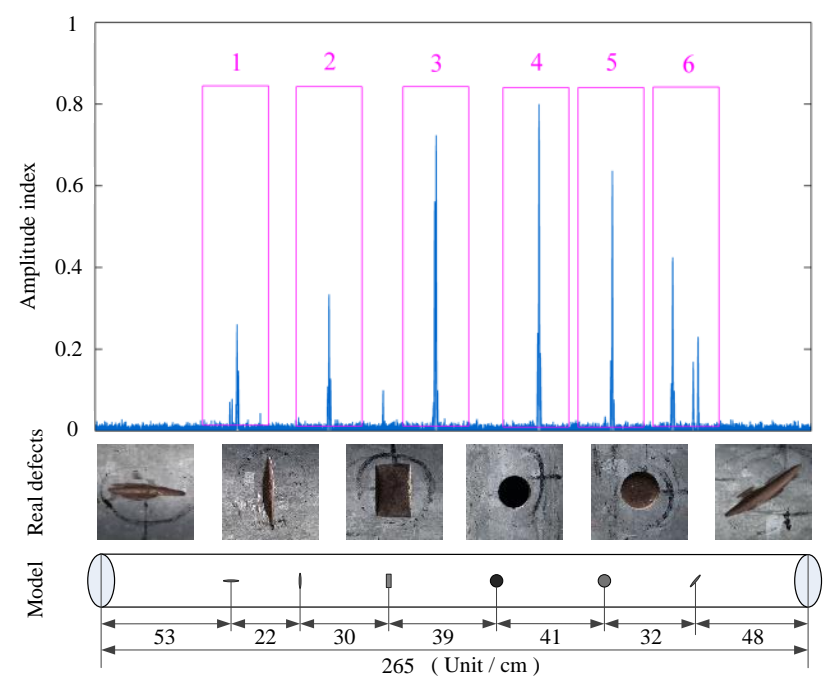

Figure 18. Experimental result

\section{CONCLUSIONS}

A harmonic detection method for the defects of metal pipeline is proposed in this paper. High sensitivity and spatial resolution are obtained by an excitation focusing array and a TMR multi-dimensional differential sensor array. A digital adjustable harmonic excitation source based on ARM CortexM3 is developed, its total power can achieve more than $70 \mathrm{~W}$, and the output signal parameters can display in 3.5-inch LCD. A high-speed data acquisition system with dual controllers based on FPGA and ARM Cortex-M4 is developed. A 7-inch touch screen can display the XYZ three-axis curve in real time, and it is equipped with GPS positioning and USB flash disk 
local storage functions in this system. The real-time parallel storage rate of 15 channels can up to $4.7 \mathrm{kHz}$ each channel. The EMD and variable scale SR algorithm for pipeline defects signal extraction is proposed, which can improve the SNR, RSME and KF effectively.

The simulation and practical experiments show that the focusing array detector is reasonable and effective, the harmonic excitation source can output stably, the high-speed acquisition and storage system can record signals completely, and the signal processing algorithm can identify the location of the pipeline defects accurately. The whole system has the characteristics of low cost, small volume and simple operation, which is more suitable to be popularized in engineering detection. At the same time, this method and instrument provide a novel idea for the detection of metal material damage with certain thickness.

In the future, we will integrate the excitation source, acquisition instrument and li-battery together and install them on a small inspection vehicle, which is easy to move and realize automatic detection. A large number of experiments will be carried out to establish a database of different types of pipeline defects, and further research will continue in terms of artificial neural networks and machine learning to support defect type identification, classification and credibility analysis, etc., so as to achieve intelligent detection and more effectively guarantee the safe operation of pipelines.

\section{ACKNOWLEDGMENT}

This work is supported by the National Key Research and Development Program of China (Grant numbers: 2017YFC0805005-1) and the Science and Technology Program of Beijing Municipal Education Commission (Grant numbers: KZ201810005009).

\section{REFERENCES}

[1] Gou, R.B., Dan, W.J., Qiu, F., Yu, M., Zhang, W.G. (2015). Welding residual stress measurement of an urban buried gas pipeline by X-ray diffraction method. Insight: Non-Destructive Testing \& Condition Monitoring, 57(10):

556-561. https://doi.org/10.1784/insi.2015.57.10.556

[2] Chang, M.K., Sun, H.S., Ciou, J.C. (2011). Applying Ultrasonic Testing to Detect Hole Defect Near the Surface. Advanced Materials Research, 194-196: 2054 2057.

https://doi.org/10.4028/www.scientific.net/amr.194196.2054

[3] Song, S.P., Que, P.W (2006). An effective defect identification scheme in pipeline ultrasonic testing. Russian Journal of Nondestructive Testing, 42(4): 255260. https://doi.org/10.1134/s1061830906040061

[4] Dai, X., Mo, J.Q., Yin, B., Hu, W., Wang, S.G. (2013). An automatic magnetic particle inspection system for detecting defects in mooring chains. Insight: NonDestructive Testing and Condition Monitoring, 55(1): 29-34. https://doi.org/10.1784/insi.2012.55.1.29

[5] Cicic, D.T., Amza, C.G., Popescu, D., Anania, F.D. (2013). Automated stand for non-destructive testing evaluation of metal products. Applied Mechanics and Materials,

371:

$401-405$ https://doi.org/10.4028/www.scientific.net/amm.371.40 1

[6] Ali, K.B., Abdalla, A.N., Rifai, D., Faraj, M.A. (2017). Review on system development in eddy current testing and technique for defect classification and characterization. IET Circuits Devices \& Systems, 11(4): 330-343. https://doi.org/10.1049/iet-cds.2016.0327

[7] Feng, B., Ribeiro, A., Rocha, T., Ramos, H. (2018). Comparison of inspecting non-ferromagnetic and ferromagnetic metals using velocity induced eddy current probe. Sensors, 18(10): 3199. https://doi.org/10.3390/s18103199

[8] Liu, B., He, L.Y., Zhang, H., Cao, Y., Fernandes, H. (2017). The axial crack testing model for long distance oil-gas pipeline based on magnetic flux leakage internal inspection method. Measurement, 103: 275-282. https://doi.org/10.1016/j.measurement.2017.02.051

[9] Hu, B., Yu, R.Q., Liu, J. (2016). Experimental study on the corrosion testing of a buried metal pipeline by transient electromagnetic method. Anti-Corros. Methods Mater, 63(4): 262-268. https://doi.org/10.1108/acmm10-2014-1444

[10] Liao, K.X., Quan, K.Y., Chun, Z. (2011). Principle and technical characteristics of non-contact magnetic tomography method inspection for oil and gas pipeline. ICPTT, Sustainable Solutions for Water, Sewer, Gas, and Oil Pipelines, 1039-1048. https://doi.org/10.1061/41202(423)111

[11] Zhang, T., Wang, X.H., Chen, Y.C., Ullah, Z., Ju, H.Y., Zhao, Y.Z. (2019). Non-contact geomagnetic detection using improved complete ensemble empirical mode decomposition with adaptive noise and Teager energy $\begin{array}{llll}\text { operator. } & \text { Electronics, } & 8(3) \text { : } & \end{array}$ https://doi.org/10.3390/electronics8030309

[12] Krivoi, G.S. (2008). NoPig: An above-ground inspection technique for non-Piggable pipelines. Oil. Gas European Magazine, 34(3): 122-124.

[13] Muñoz, C.Q.H., García, M.F.P., Benjamin, L., Alfredo, A. (2017). New pipe notch detection and location method for short distances employing ultrasonic guided waves. Acta Acustica United with Acustica, 103(5): 772-781. https://doi.org/10.3813/aaa.919106

[14] Ma, Z.L., Liu, H.D. (2011). Temperature error compensation new method of MFL sensor to oil-gas pipeline corrosion inspection. Advanced Materials Research, 204-210: 1026-1030. https://doi.org/10.4028/www.scientific.net/amr.204210.1026

[15] Zarifi, M.H., Deif, S., Abdolrazzaghi, M. et al. (2017). A microwave ring resonator sensor for early detection of breaches in pipeline coatings. IEEE Transactions on Industrial Electronics, 65(2): 1626-1635. https://doi.org/10.1109/tie.2017.2733449

[16] Dimitropoulos, P.D., Avaritsiotis, J.N. (2003). Integrating the Fluxgate principle in the Spin-Valve and AMR sensor technologies. Sensors \& Actuators A: Physical, 106(1-3): 43-47. https://doi.org/10.1016/s0924-4247(03)00130-4

[17] Vacher, F., Alves, F., Gilles-Pascaud, C. (2007). Eddy current nondestructive testing with giant magnetoimpedance sensor. NDT \& E International, 40(6): 439442. https://doi.org/10.1016/j.ndteint.2007.02.002

[18] Wu, B., Wang, Y.J., Liu, X.C., He, C.F. (2015). A novel TMR-based MFL sensor for steel wire rope inspection 
using the orthogonal test method. Smart Materials and Structures, 24(7): 075007. https://doi.org/10.1088/09641726/24/7/075007

[19] Kreutzbruck, M.V., Allweins, K., Ruhl, T., Muck, M., Heiden, C., Krause, H.J., Hohmann, R. (2011). Defect detection and classification using a SQUID based multiple frequency eddy current NDE system. IEEE Transactions on Applied Superconductivity, 11(1): 1032-1037. https://doi.org/10.1109/77.919525

[20] Li, W., He, Z., Knoll, G.F., Wehe, D.K., Berry, J.E. (2000). A data acquisition and processing system for 3D position sensitive CZT gamma-ray spectrometers. IEEE Transactions on Nuclear Science, 46(6): 1989-1994. https://doi.org/10.1109/23.819268

[21] Vasil'ev, A.E., Kolodeznikov, I.V. (2015). The development and use of data acquisition and control systems based on arm microcontrollers. Measurement Techniques, 58(3): 245-249. https://doi.org/10.1007/s11018-015-0693-3

[22] Xue, T., Zhu, J.F., Gong, G.H., et al. (2018) The design and data-throughput performance of readout module based on ZYNQ SoC. IEEE Transactions on Nuclear Science, $\quad 65(5)$ : $1169-1179$ https://doi.org/10.1109/tns.2018.2820706

[23] Li, H.R., Pang, L.J., Zhang, X.F., Deng, G., Tong, Y.W. (2011). The application of FPGA and DSP techniques in intelligent wattmeter. Advanced Materials Research, 201-203. 2096-2100. https://doi.org/10.4028/www.scientific.net/amr.201203.2096

[24] Da Silva, B., Segers, L., Braeken, A., et al. (2019). Design exploration and performance strategies towards power-efficient FPGA-based architectures for sound source localization. Journal of Sensors, 2019: 5761235. https://doi.org/10.1155/2019/5761235

[25] Bosse, J., Joubert, P.Y., Larzabal, P., Ferréol, A. (2010). High resolution approach for the localization of buried defects in the multi-frequency eddy current imaging of metallic structures. NDT \& E International, 43(3): 250257. https://doi.org/10.1016/j.ndteint.2009.12.002
[26] Huang, Y.M., Wu, D., Zhang, Z.F., Chen, H.B., Chen, S.B. (2017). EMD-based pulsed TIG welding process porosity defect detection and defect diagnosis using GASVM. Journal of Materials Processing Technology, 239: 92-102. https://doi.org/10.1016/j.jmatprotec.2016.07.015

[27] Song, Q., Ding, W.X., Peng, H., Gu, J., Shuai, J. (2017). Pipe defect detection with remote magnetic inspection and wavelet analysis. Wireless Personal Communications, $\quad$ 95(3): 2299-2313. https://doi.org/10.1007/s11277-017-4092-8

[28] Wan, C.B., Pan, M.C., Zhang, Q., Wu, F.H., Pan, L., Sun, X.Y. (2018). Magnetic anomaly detection based on stochastic resonance. Sensors and Actuators A: Physical, 278: 11-17. https://doi.org/10.1016/j.sna.2018.05.009

[29] Fartookzadeh, M. (2019). Serial-fed linear frequency diverse arrays for obtaining direction of arrival (DOA) of frequency modulated continuous waveform (FMCW) sources with ambiguity reduction. International Journal of RF and Microwave Computer-Aided Engineering, 29(10): e21900. https://doi.org/10.1002/mmce. 21900

[30] Kapisch, E.B., Silva, L.R.M., Martins, C.H.N., et al. (2016). An implementation of a Power System Smart Waveform Recorder using FPGA and ARM cores. Measurement, 90: 372-381. https://doi.org/10.1016/j.measurement.2016.04.062

[31] Pierleoni, P., Palma, L., Belli, A., et al. (2019). An EMDbased algorithm for emboli detection in echo Doppler audio signals. Electronics, 8(8): 824. https://doi.org/10.3390/electronics8080824

[32] Shi, P.M., Yuan, D.Z., Han, D.Y., et al. (2018). Stochastic resonance in a time-delayed feedback tristable system and its application in fault diagnosis. Journal of Sound and Vibration, 424: 1-14. https://doi.org/10.1016/j.jsv.2018.03.007

[33] Liu, J.J., Leng, Y.G., Lai, Z.H., Fan, S.B. (2018). Multifrequency signal detection based on frequency exchange and re-scaling stochastic resonance and its application to weak fault diagnosis. Sensors, 18(5): 1325. https://doi.org/10.3390/s18051325 